\title{
A New Design of Pressure Sensor Based on a GaAs- based Two Dimensional Photonic Crystal Slab on SiO2 Substrate
}

\author{
Saeide Norouzi \\ Golestan University \\ kiazand fasihi ( $\sim$ k.fasihi@gu.ac.ir) \\ Golestan University https://orcid.org/0000-0001-9918-6201
}

\section{Research Article}

Keywords: deformation, photonic crystal (PC), pressure sensor, quality factor, refractive index

Posted Date: November 5th, 2021

DOl: https://doi.org/10.21203/rs.3.rs-985138/v1

License: (c) (1) This work is licensed under a Creative Commons Attribution 4.0 International License.

Read Full License 


\title{
A new design of pressure sensor based on a GaAs-based two dimensional photonic crystal slab on $\mathrm{SiO}_{2}$ substrate
}

\author{
Saeide Norouzi and Kiazand Fasihi* \\ Department of Electrical Engineering, Faculty of Engineering, Golestan University, Gorgan, Iran \\ s71.norouzi@gmail.com, k.fasihi@gu.ac.ir
}

\begin{abstract}
This paper reports the design and three dimensional (3D) simulation of a new photonic crystal (PC) pressure sensor. The device is constructed using a GaAs-based 2D PC slab on $\mathrm{SiO}_{2}$ substrate. The strain/stress simulations and also the spectral simulations are done using CST Studio Suite. In this investigation, the sensitivity of the proposed pressure sensor is calculated by considering the variation of the refractive index and also the deformation of the structure. The numerical results show that when pressure is applied, the refractive index variations cause an increment in the resonant wavelength of the transmission spectrum, but the deformation factor cause a decrease in the resonant wavelength. It has been shown that the relationship between the applied pressure and the resonant wavelength of the designed micro-cavity is linear. Based on the simulation results, the quality factor of the designed micro-cavity and the sensitivity of the pressure sensor are 2200 and $3.5 \mathrm{~nm} / \mathrm{GPa}$, respectively.
\end{abstract}

Index Terms- deformation; photonic crystal $\quad(\mathrm{PC})$; pressure sensor; quality factor; refractive index.

\section{Introduction}

The photonic crystals (PCs) that are periodic structures (with a period comparable to the wavelength of the light) of dielectric materials, have attained growing attention due to their outstanding applications in the photonics [1-4]. These attentions are mainly due to the existence of photonic band-gap, which presents the unique capability to control the propagation of the light. The means to control the propagation of the light is principally obtained by introducing defects in PCs. In PC-based structures, the micro-cavities are formed by point defects and waveguides are formed by line defects. The PC micro-cavities, owing to micro-sizes and high quality factors are excellent candidates to obtain photonic sensors [5-10].

As we know, the PCs can be presented in one, two, or three dimensional formations. Furthermore, the frequency domain of photonic band-gap of PCs can be determined by the geometry and the refractive index of the constituent material [11]. By introducing any kind of defect, the periodicity of the PC lattice will be destroyed; and one or more resonant modes may be created in the photonic band-gap. The point defects in PCs can be made by changing the location of one or more holes (or rods), by reducing or increasing the radii, or even by removing them. A point defect embedded in the PC structure, that acts as a micro-cavity, can trap the light. So far, a few PC-based structures have been suggested for strain/stress [12-15], and pressure sensor [16-24].

In 2004, Radanovich et al. [14] presented a 2D PC sensor for mid-infrared range with incorporated a 90-degree bend waveguide, and investigated the influence of stress on electromagnetic wave propagation in such structures, utilized 2D mechanical and electromagnetic finite element modeling. In 2010, Tung et al. [15] using finite element method (FEM) analysis and 2D FDTD simulation investigated the strain sensitivity of the resonant wavelength of Si-PC micro-cavity resonators. In 2007, Stomeo et al. [16] realized a force/pressure optical sensor by designing a bulk GaAs/AlGaAs photonic crystal microcavity operating in the wavelength range 1300-1400 nm. In 2007, Biallo et al. [17] proposed a 2D PC microcavity (designed on a GaAs membrane) coupled to a PC waveguide to realise a highly sensitive pressure sensor and evaluated the change of the refractive index induced by the application of a force onto a sensing surface. In 2011, Tung et al. [18] presented the theoretical and experimental investigations on the strain sensing effects of a 2D PC micro-cavity. They only investigated the influence of strain to the geometry of the holes lattice in the Si slab. Furthermore, they reported that in their structure the stress/strains and consequently the refractive index of material was distributed non-uniformly, which was not included in the simulation. This issue made the simulated and measured results to be inconsistent. In 2011, Lu et al. [19] implemented an all-optical pressure sensor using controllable vertical silicon nanowire arrays that are on a $\mathrm{Si} / \mathrm{SiO}_{2} \mathrm{membrane}$. When hydrostatic pressure is applied the membrane is bent, as a result, the color of the membrane is changed on account of the modulation of the nanowire pitch and deflection angle. In 2012, Olyaee et al. [20] proposed a 2D PC pressure sensor that was based on a nanocavity that was side coupled to a waveguide. In 2014, Shanthi et al. [21] proposed a 2D PC pressure sensor that consists of two PC quasi waveguides and L3 defect that is placed in between the two quasi waveguides. In 2016, Tao et al. [22] proposed a 2D PC pressure sensor that consists of two PC quasi waveguides and an optimized nanocavity that is placed in between the two quasi waveguides. In 2017, Wong et al. [23] realized and simulated (using FEM analysis and 
also rigorous coupled-wave analysis) a pressure sensor using PC mirror pressure-sensing diaphragms with silicon-on-nothing (SON) reference cavities. In 2021, Mishra et al. [24] proposed a 1D indium arsenide (InAs) PC-waveguide-based pressure sensor with respect to the output transmitted intensity in 3-communication windows.

In this paper, the design and 3D simulation of a new PC pressure sensor is presented. The device is constructed using a micro-cavity embedded in GaAs-based 2D PC slab on $\mathrm{SiO}_{2}$ substrate. The simulations are based on FEM and finite-difference time-domain (FDTD) method and are done using CST Studio Suite software. The sensitivity of the proposed sensor is calculated by considering the factors of the refractive index variation and the deformation. Based on the simulation results, the quality factor of the micro-cavity and the sensitivity of the proposed sensor are 2021 and $3.5 \mathrm{~nm} / \mathrm{GPa}$, respectively. Table.1 summarizes the used structure, the simulation method, the considered factors, the sensitivity and the quality factor of the previous designs and our proposed pressure sensor. It is expected that the proposed device can find applications in photonic pressure sensing.

\section{The structure and the analysis of the proposed pressure sensor}

\subsection{The spectral specifications of the used micro-cavity}

Fig. 1 shows the structure of the proposed pressure sensor, that is based on a micro-cavity which is embedded in a GaAsbased 2D PC slab. The 2D PC slab is deposited on a substrate of $\mathrm{SiO}_{2}$. The PC is composed of a triangular lattice of air holes in a dielectric background. In the proposed structure, the refractive index of the 2D PC slab and the substrate (at the wavelength of $1.55 \mu \mathrm{m}$ ) are 3.43 and 1.5, respectively [17]. The thicknesses of the 2D PC slab and the substrate are $479.74 \mathrm{~nm}$ and $1050 \mathrm{~nm}$, respectively. The radius of the air holes is $124.4 \mathrm{~nm}$, and the lattice constant is $a=373.5 \mathrm{~nm}$. The resonant micro-cavity is constructed by filling the three middle air holes, and in order to increase the quality factor, the two marked air holes are shifted from the original lattice positions by $s=0.2 a$ (see Fig. 1). The excitations are continuous waves with a Gaussian envelope, which launch the light power into the left facet of the PC slab. The output power is measured at the right facet of the PC slab. Here, the 3D FDTD simulations are for transverse-electric (TE) polarization and are done using CST Microwave Studio software. The transmission and reflection spectra of the resonant mode of the designed micro-cavity are shown in Fig. 2. As can be seen, the PC exhibits a band-gap for wavelengths between $1055 \mathrm{~nm}$ and $1570 \mathrm{~nm}$. Table. 2 shows the spectral specifications of the used micro-cavity for different values of $s$ (the shift of the marked air holes from the original lattice positions, see Fig. 1) parameter. As can be seen, when the applied pressure is zero, there is a resonant wavelength of $\lambda_{\text {res } 0}=1540.7$ with the quality factor and the transmission of 2200 and 0.005 , respectively.

\subsection{The pressure sensor analysis, considering only the refractive index variation}

As mentioned previously, the optical properties of materials, such as their refractive index, can be changed by mechanical stress/strain. The refractive index changes due to stress in an arbitrary direction, can be expressed by the stress-optical tensor equation [25]

$$
\left(\begin{array}{l}
n_{x x} \\
n_{y y} \\
n_{z z} \\
n_{y z} \\
n_{x z} \\
n_{x y}
\end{array}\right)=\left(\begin{array}{l}
n_{0} \\
n_{0} \\
n_{0} \\
0 \\
0 \\
0
\end{array}\right)-\left(\begin{array}{cccccc}
C_{1} & C_{2} & C_{2} & 0 & 0 & 0 \\
C_{2} & C_{1} & C_{2} & 0 & 0 & 0 \\
C_{2} & C_{2} & C_{1} & 0 & 0 & 0 \\
0 & 0 & 0 & C_{3} & 0 & 0 \\
0 & 0 & 0 & 0 & C_{3} & 0 \\
0 & 0 & 0 & 0 & 0 & C_{3}
\end{array}\right)\left(\begin{array}{l}
\sigma_{x x} \\
\sigma_{y y} \\
\sigma_{z z} \\
\sigma_{y z} \\
\sigma_{x z} \\
\sigma_{x y}
\end{array}\right),
$$

where $\sigma_{i j}$ and $n_{i j}$ are the stress and the refractive index in the $i j$ direction, respectively.-refractive index in stress is the $n_{0}$ free state, and $C_{1}, C_{2}$ and $C_{3}$ are the stress-optic constants that can be calculated as

$$
\begin{gathered}
C_{1}=\frac{n_{0}^{3}\left(p_{11}-2 v p_{12}\right)}{(2 E)}, \\
C_{2}=\frac{n_{0}^{3}\left(p_{12}-v\left(p_{11}+p_{12}\right)\right)}{(2 E)}, \\
C_{3}=\frac{n_{0}^{3} p_{44}}{(2 G)},
\end{gathered}
$$

where $p_{11}, p_{12}$ and $p_{44}$ are the strain-optic constants. G, E, and $v$ are the shear modulus, the Young's modulus and the Poisson's ratio, respectively [24]. Note that because the proposed PC pressure sensor constructed by etching several air holes in the GaAs slab, for a non vertical pressure, the strain/stress components in the GaAs slab are distributed non-uniformly. In 
this case, the simulation of the proposed structure for the non-uniformly distributed anisotropic refractive index is too complicated. For this reason, in this paper, only a pressure in the vertical direction (perpendicular to the incident plane), is applied to the structure (the bottom facet of the structure is assumed to be fixed). As a result, for the proposed structure undergone the vertical pressure (as shown in Fig.1), only the $\sigma_{z z}$ component is non-zero (and can be considered to be uniform through the thickness), and $\sigma_{x x}=\sigma_{y y}=\sigma_{y z}=\sigma_{x z}=\sigma_{x y}=0$. In this case, considering the (1), we have

$$
\begin{aligned}
& n_{x x}=n_{0}-C_{2} \sigma_{z z}, \\
& n_{y y}=n_{0}-C_{2} \sigma_{z z}, \\
& n_{z z}=n_{0}-C_{1} \sigma_{z z} .
\end{aligned}
$$

For GaAs (at the wavelength of $\lambda=1.15 \mu \mathrm{m}$ ), $p_{11}=-0.165$ and $p_{12}=-0.14$ [25]. In this case from (2) and (3), we have $C_{1}=-17.01 \times 10^{-12} / \mathrm{Pa}$ and $C_{2}=-11.61 \times 10^{-12} / \mathrm{Pa}$, and as a result

$$
\begin{aligned}
& n_{x x}=n_{0}+\left(11.61 \times 10^{-12} \times \sigma_{z z}\right), \\
& n_{y y}=n_{0}+\left(11.61 \times 10^{-12} \times \sigma_{z z}\right), \\
& n_{z z}=n_{0}+\left(17.01 \times 10^{-12} \times \sigma_{z z}\right) .
\end{aligned}
$$

The used parameter of the shear modulus, the Young's modulus and the Poisson's ratio, for the $\mathrm{GaAs}\left(\mathrm{SiO}_{2}\right)$ are $116 \mathrm{GPa}, 0.24$ and 0.24 (66.3 GPa, 0.15 and 0.24$)$, respectively [26]. Using (8)-(10) the pressure-modified refractive index values are calculated for different values of pressure and shown in Table. 3. The values of the stress $\left(\sigma_{z z}\right)$ are obtained by numerical simulation of the proposed structure using the CST MPhysics Studio software. The stress distribution due to applying the $2 \mathrm{GPa}$ pressure, perpendicular to the plane of incidence, is shown in Fig. 3. As can be seen, the stress is distributed uniformly in the GaAs slab.

In the following, the resonant spectrum of the designed micro-cavity is investigated considering the effect of the pressuremodified refractive index variations. Fig. 4 shows the 3D FDTD simulation results of the transmission spectrum for the different values of the applied vertical pressure. As can be seen, when the applied pressure is increased, the resonant wavelength is shifted toward higher wavelengths. Fig. 5 shows that a linear dependence is observed between the resonant wavelength and the pressure. From this figure the sensitivity of the proposed pressure sensor, considering the effect of the pressure-modified refractive index variations, can be calculated as

$$
S_{r}=\frac{\Delta \lambda}{\Delta P}=\frac{1550.7-1540.7}{2-0}=5 \mathrm{~nm} / \mathrm{GPa}
$$

\subsection{The pressure sensor analysis, considering only the deformation factor}

In the general case, for a non vertical pressure, the geometry of a PC structure, including the positions and the shapes of air holes is changed. But in our design, when a vertical pressure is applied (perpendicular to the incident planewhen and ( $\left.-\hat{a}_{z}\right)$, the applied pressure is below $4 G P a$ ), the strain/stress is distributed uniformly in the structure. In this case, only the thickness of the structure is changed and the radius of the air holes are remains almost unchanged. The values of the strain, $\gamma$, are obtained by numerical simulation of the proposed structure using the CST MPhysics Studio software. The deformation of the proposed structure due to applying the $4 G P a$ pressure, perpendicular to the plane of incidence, is shown in Fig. 6 . As can be seen, the stress is distributed uniformly in the GaAs slab. Using the strain definition [27], the variation of the thickness of the PC GaAs slab, $\Delta d$, can be calculated from

$$
\Delta d=\gamma d_{0}
$$

where $d_{0}$ is the initial value of the thickness of the slab. Table. 4. shows the strain, the thickness variation, and the new thickness of the GaAs slab, for different values of the vertical pressure.

The 3D FDTD simulation results of the transmission spectra, considering the deformation effect, for the different values of the applied vertical pressure is shown in Fig. 7. In this case, one can see that when the applied pressure is increased, the resonant wavelength is shifted toward lower wavelengths and a linear dependence is observed between the resonant wavelength and the pressure (see Fig. 8). Furthermore, in this case, the sensitivity can be calculated as 


$$
S_{d}=\frac{\Delta \lambda}{\Delta P}=\frac{1537.7-1540.7}{2-0}=-1.5 \mathrm{~nm} / \mathrm{GPa}
$$

By comparing the (11) and (13), it can be deduced that the refractive index variations have a greater effect on the sensitivity than the deformation. Furthermore, as can be seen, these effects are in opposite directions.

\subsection{The pressure sensor specification, considering both the refractive index variation and the deformation}

Now, we investigate the simultaneous effect of both factors on the performance of the device. In this case, for a given pressure, the values of the refractive index and the thickness of the GaAs slab are obtained from Tables 3 and 4, respectively. In this case, the transmission spectra of the proposed pressure sensor for the different value of the applied vertical pressure is shown in Fig. 9. From Fig. 10 one can see that when the applied pressure is increased, the resonant wavelength is linearly shifted toward higher wavelengths. Furthermore, from Fig. 10, the sensitivity of the proposed pressure sensor, considering both effects, can be calculated as

$$
S_{t}=\frac{\Delta \lambda}{\Delta P}=\frac{1547.7-1540.7}{2-0}=3.5 \mathrm{~nm} / \mathrm{GPa}
$$

Overall, the numerical simulations show that both investigated factors (the refractive index variations and the deformation), have a considerable effect on the pressure sensitivity, and ignoring the impact of either factor will lead to significant error.

\section{Conclusions}

A new pressure sensor, which was based on a 2D PC GaAs slab on $\mathrm{SiO}_{2}$ substrate has been proposed and investigated using 3D FEM and 3D FDTD methods. It was shown that in the proposed device, the variation of the refractive index and also the deformation of the structure, have a considerable effect on the sensitivity. Based on the simulation results, when pressure is applied, the refractive index variations (the deformation factor) cause an increment (a decrease) in the resonant wavelength of the transmission spectrum. In summary, the simulation results show that the proposed sensor, owing to the quality factor of 2200 and the sensitivity of $3.5 \mathrm{~nm} / \mathrm{GPa}$, can be used effectively in pressure sensing applications.

\section{References}

[1] J.D. Joannopoulos, S.G. Johnson, J.N. Winn, R.D. Meade, Photonic Crystal: Molding the Flow of Light. Princeton, Princeton Univ. Press, 2008.

[2] B. Troia, A. Paolicelli, F.D. Leonardis, V.M.N. Passaro, "Photonic Crystals for Optical Sensing : A Review," Advances in Photonic Crystals, V.M.N Passaro, Ed. InTech, pp. 241-295, 2013.

[3] R.V. Nair, R. Vijaya, "Photonic crystal sensors: An overview”, Prog. Quant. Electron., 34, pp. 89-134, 2010.

[4] K. Fasihi, S. Bashiri, "A new $2 \times 1$ photonic crystal multiplexer assisted by Fano resonances and Kerr nonlinear effect," Photonics Nanostructures: Fundam. Appl, 42, 100837, 2020.

[5] A. Anamoradi, K. Fasihi, "A highly sensitive optofluidic-gas sensor using two dimensional photonic crystals," Superlattices Microstruct., 125, pp. 302309, 2019.

[6] Y. Zhang, Y. Zhao, Q. Wang, "Measurement of methane concentration with cryptophane E infiltrated photonic crystal microcavity," Sens. Actuators, B., 209, pp. 431-437, 2015.

[7] Y. Zhanga, Y. Zhaoa, R. Lv, "A review for optical sensors based on photonic crystal cavities," Sens. Actuator, A., 233, pp. 374-389, 2015.

[8] T. Tung, "sensitive effect in a triangular lattice photonic crystal hole-modified nanocavity," IEEE Sens. J., 11(11), pp. 2657-2663, 2011.

[9] Y. Yang, D. Yang, H. Tian, Y. Jia, "Photonic crystal stress sensor with high sensitivity in double directions based on shoulder-coupled aslant nanocavity," Sens. Actuators, A., 193(7), pp. 149-154, 2013.

[10] K. Maeno, S. Aki, K. Sueyoshi, H. Hisamoto, T. Endo, "Polymer-based photonic crystal cavity sensor for optical detection in the visible wavelength region," Anal Sci., 32(1), pp. 117-120, 2016.

[11] P.R. Villeneuve, S. Fan, J.D. Joannopoulos, "Microcavities in photonic crystals: Mode symmetry, tunability, and coupling efficiency," Phys. Rev., 54, pp. 7837-7842, 1996.

[12] K. Radanovich, Z. Jaksic, Z. Djuric, "Design and simulation of stressed 2D photonic crystals by finite element method", II International Symposium Light Metals and Composite Materials, 2004.

[13] Y. Yang, D. Yang, H. Tian, Y. Ji, "Photonic crystal stress sensor with high sensitivity in double directions based on shoulder-coupled aslant nanocavity," Sens. Actuators, A, 193, pp. 149-154, 2013

[14] B.T. Tung, D.V. Dao, S. Susumu, H.M. Nguyen, S. Rogge, H.W.M. Salemink, "Strain Sensitivity of a Modified Single-Defect Photonic Crystal Nanocavity for Mechanical Sensing," IEEE sensors conference, Waikoloa, USA, 2010.

[15] T.W. Lu, C.C. Wu, P.T. Lee, “1D photonic crystal strain sensors,” ACS Photonics, 5, pp. 2767-2772, 2018.

[16] T. Stomeo, M. Grande, A. Qualtieri, A. Passaseo, A. Salhi, M. De Vittorio, D. Biallo, A. D’orazio, M. De Sario, V. Marrocco, V. Petruzzelli, F. Prudenzano, "Fabrication of force sensors based on two-dimensional photonic crystal technology," Microelectron. Eng., 84, pp. $1450-1453,2007$.

[17] D. Biallo, M.D. Sario, A. D'Orazio, V. Marrocco, V. Petruzzelli, M.A. Vincenti, F. Prudenzano, T. Stomeo, M. Grande, G. Visimberga, R. Cingolani, M.D. Vittorio, "High sensitivity photonic crystal pressure sensor,” J. Eur. Opt. Soc., 2, 07017, 2007. 
[18] B.T. Tung, D.V. Dao, T. Ikeda, Y. Kanamori, K. Hane, S. Sugiyama, "Investigation of strain sensing effect in modified single-defect photonic crystal nanocavity," Opt. Express., 19(9), pp. 8821-8829, 2011

[19] Y. Lu, A. Lal, "Photonic crystal based all-optical pressure sensor," IEEE conference, MEMS, Cancun, Mexico, pp. 621-624, 2011.

[20] S. Olyaee, A.A. D Dehghani "High resolution and wide dynamic range pressure sensor based on two-dimensional photonic crystal," Photonic Sens, 2(1), pp. 92-96, 2012

[21] K.V. Shanthi, S. Robinson, "Two-Dimensional photonic crystal based sensor for pressure sensing," Photonic Sens, 4(3), pp. 248-253, 2014.

[22] S. Tao, D. Chen, J. Wang, J. Qiao, Y. Duan, "A high sensitivity pressure sensor based on two-dimensional photonic crystal," Photonic Sens, 6(2), pp. $137-142,2016$.

[23] Y.P. Wong, J. Bregman, O. Solgaard, "Monolithic silicon-on-nothing photonic crystal pressure sensor,” IEEE conference, Transducers, Kaohsiung, Taiwan, pp. 1963-1966, 2017.

[24] C.S. Mishra, M.R. Nayak, R. Arunachalam, G.Palai, "Realisation of pressure sensor using one dimensional indium arsenide (InAs) based photonic waveguide in 3-communication windows," IEEE conference, Devices for Integrated Circuit (DevIC), Kalyani, India, pp. 1-18, 2021.

[25] M. Huang, "Stress effects on the performance of optical waveguides," Int. J. Solids Struct., 40, pp. 1615-1632, 2003.

[26] M.J. Weber, Handbook of Optical Materials, (CRC Press LLC, Boca Raton, 2003).

[27] F.B. Beer, Jr.E. Russell Johnston, J.T. DeWolf, D.F. Mazurek, Mechanics of Materials. 7th ed. McGraw-Hill Education, 2014. 


\section{Dear Editor}

Hereby, the authors confirm that the article:

"A new design of pressure sensor based on a GaAs-based two dimensional photonic crystal slab on $\mathrm{SiO}_{2}$ substrate"

is original and has not been published previously or is not under consideration for publication elsewhere. We also confirm that if this article accepted, it will not be published elsewhere in the same form, in any language, without the written consent of the publisher.

Furthermore, we confirm that:

- We have not received any funding support for the investigation.

- There are not any conflicts of interest about the investigation.

Thank you.

Regards,

\section{Kiazand Fasihi}

Associate Professor in Electrical Engineering

Dept. of Electrical Engineering

Golestan University,

Gorgan, Iran

Saeide Norouzi

Dept. of Electrical Engineering

Golestan University,

Gorgan, Iran

Kiazand Fasihi was born in 1977. He received B.Sc. degree from Razi University and M.Sc. and Ph.D. degrees from Iran University of Science and Technology (IUST). From 2010, he is an Associate Professor at Golestan University, Gorgan, Iran. His research interests include optoelectronics, photonic crystal devices, plasmonics, and optical integrated circuits.

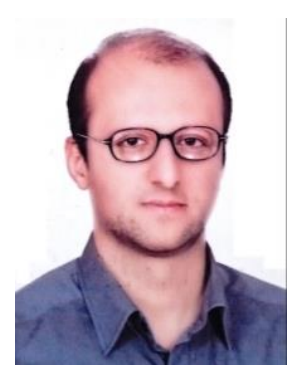

Saeide Norouzi was born in 1993. She received B.Sc. and M.Sc. degrees from Golestan University. From 2019, she is an instructor at Golestan University, Gorgan, Iran. Her research interests include photonic crystal devices.

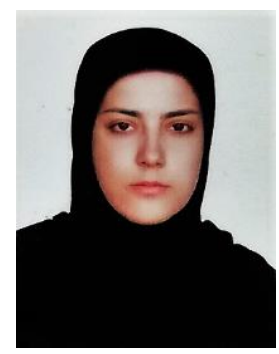




\section{Figures}

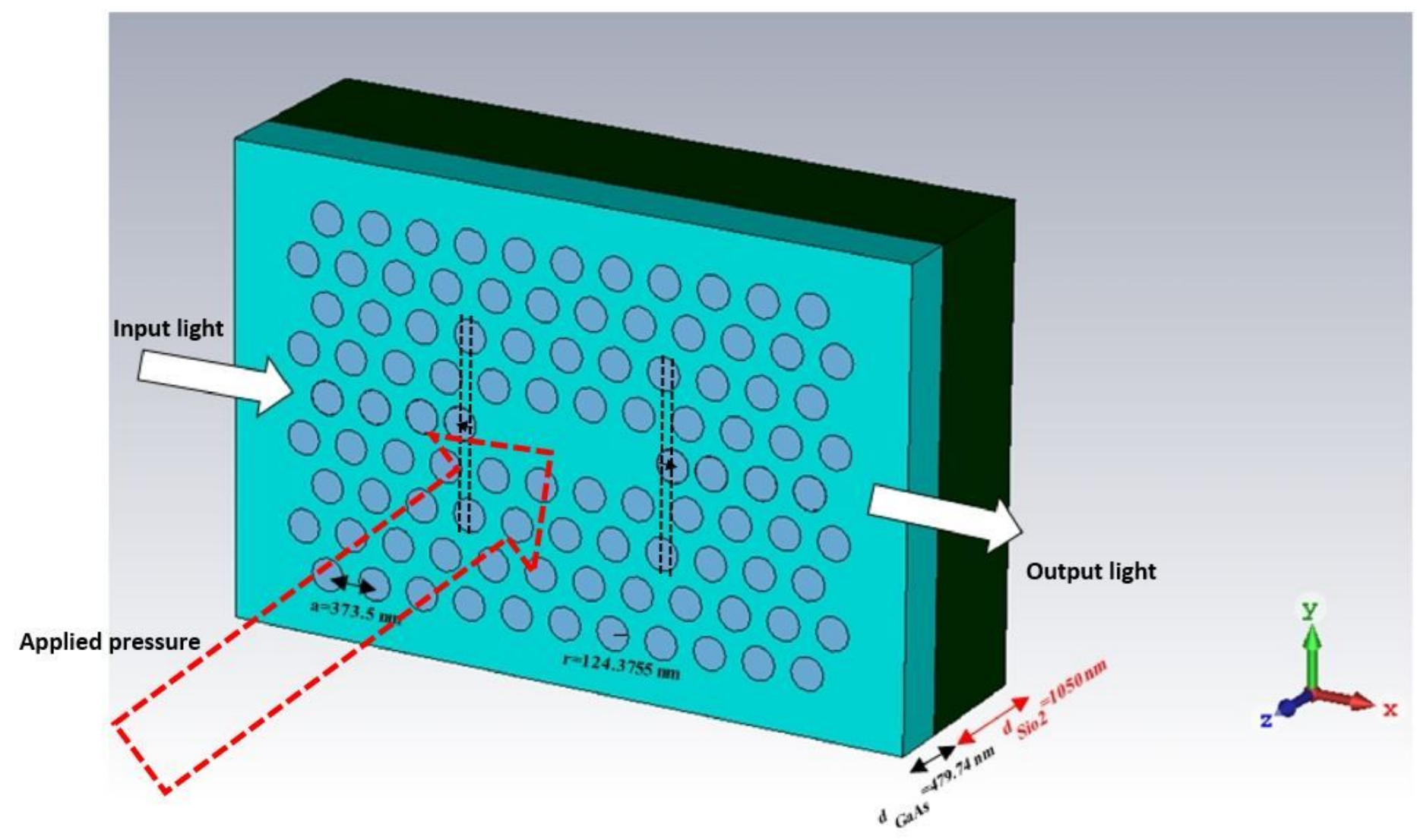

\section{Figure 1}

The structure of the proposed pressure sensor. The thicknesses of the 2D PC slab and the substrate are $479.75 \mathrm{~nm}$ and $1050 \mathrm{~nm}$, respectively. The radius of the air holes is $124.4 \mathrm{~nm}$ and the lattice constant is $a=373.5 \mathrm{~nm}$. The two marked air holes are shifted from the original lattice positions by $0.2 \mathrm{a}$. 


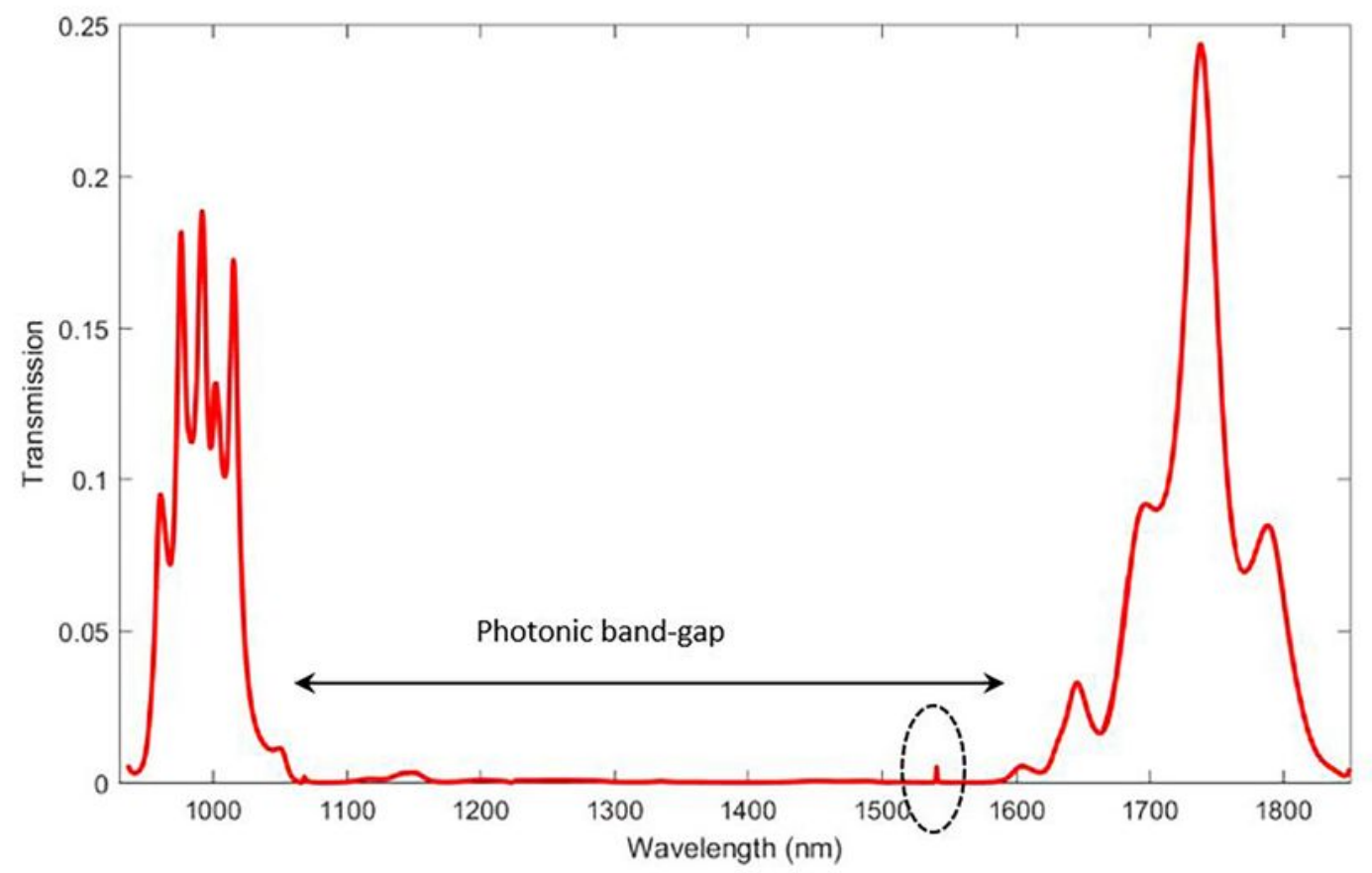

(a)

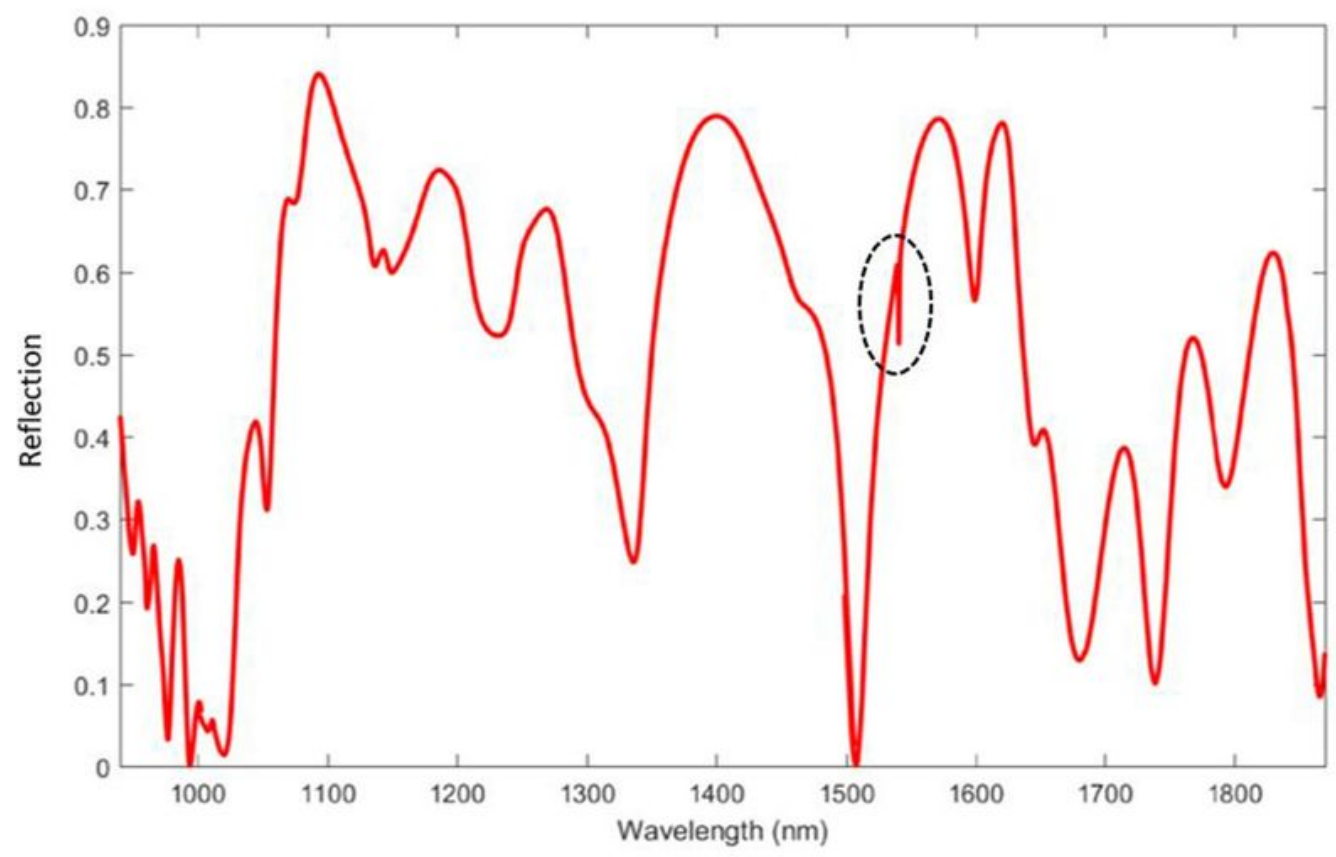

(b)

Figure 2

(a) The transmission spectrum of the proposed structure. There is a resonanse wavelength at $\lambda$ res $0=$ $1540.7 \mathrm{~nm}$, with the transmission of 0.005 , (b) The reflection spectrum of the proposed structure 

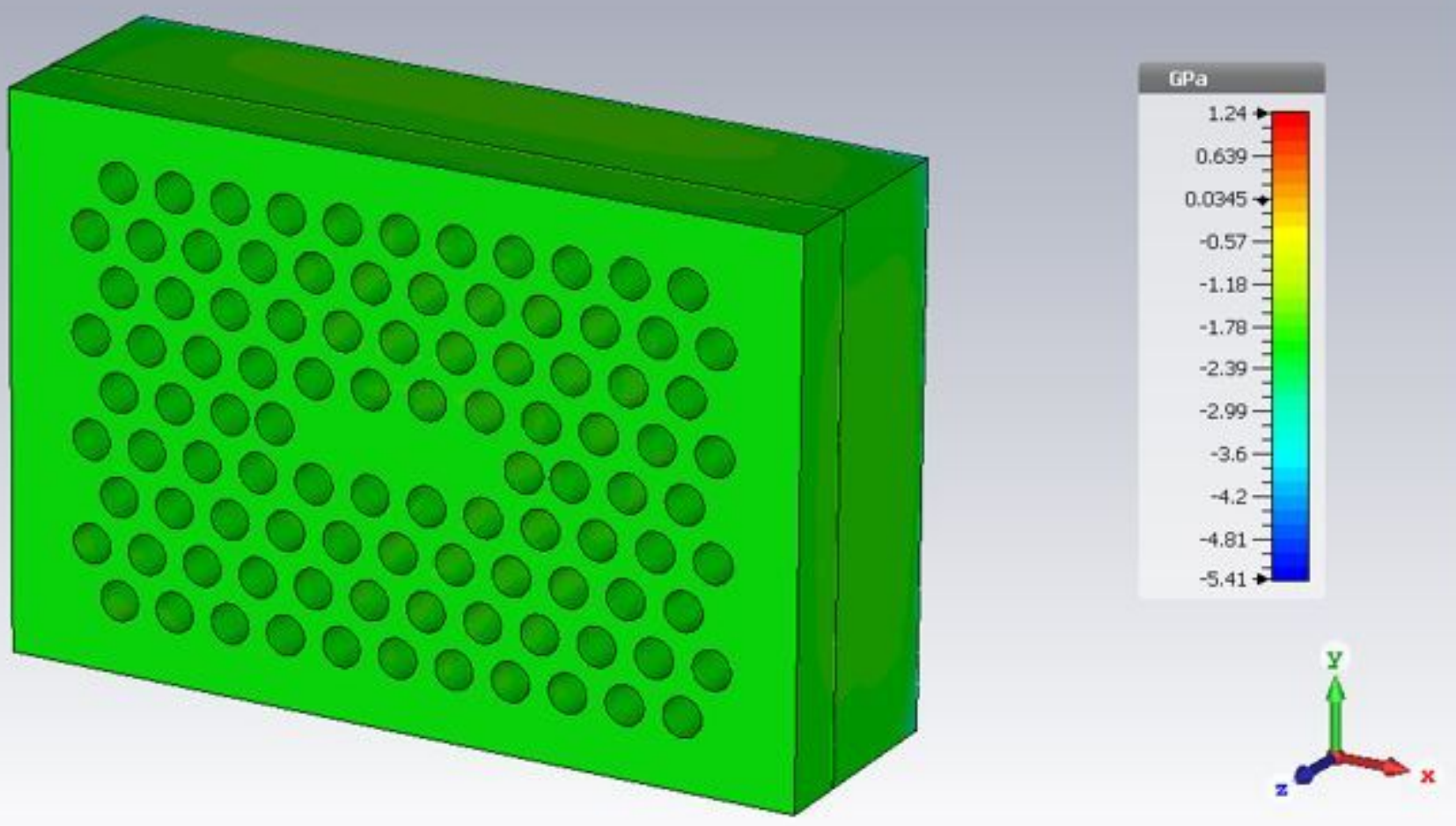

Figure 3

The stress distribution, when a pressure, perpendicular to the plane of incidence (-aa), is applied. 


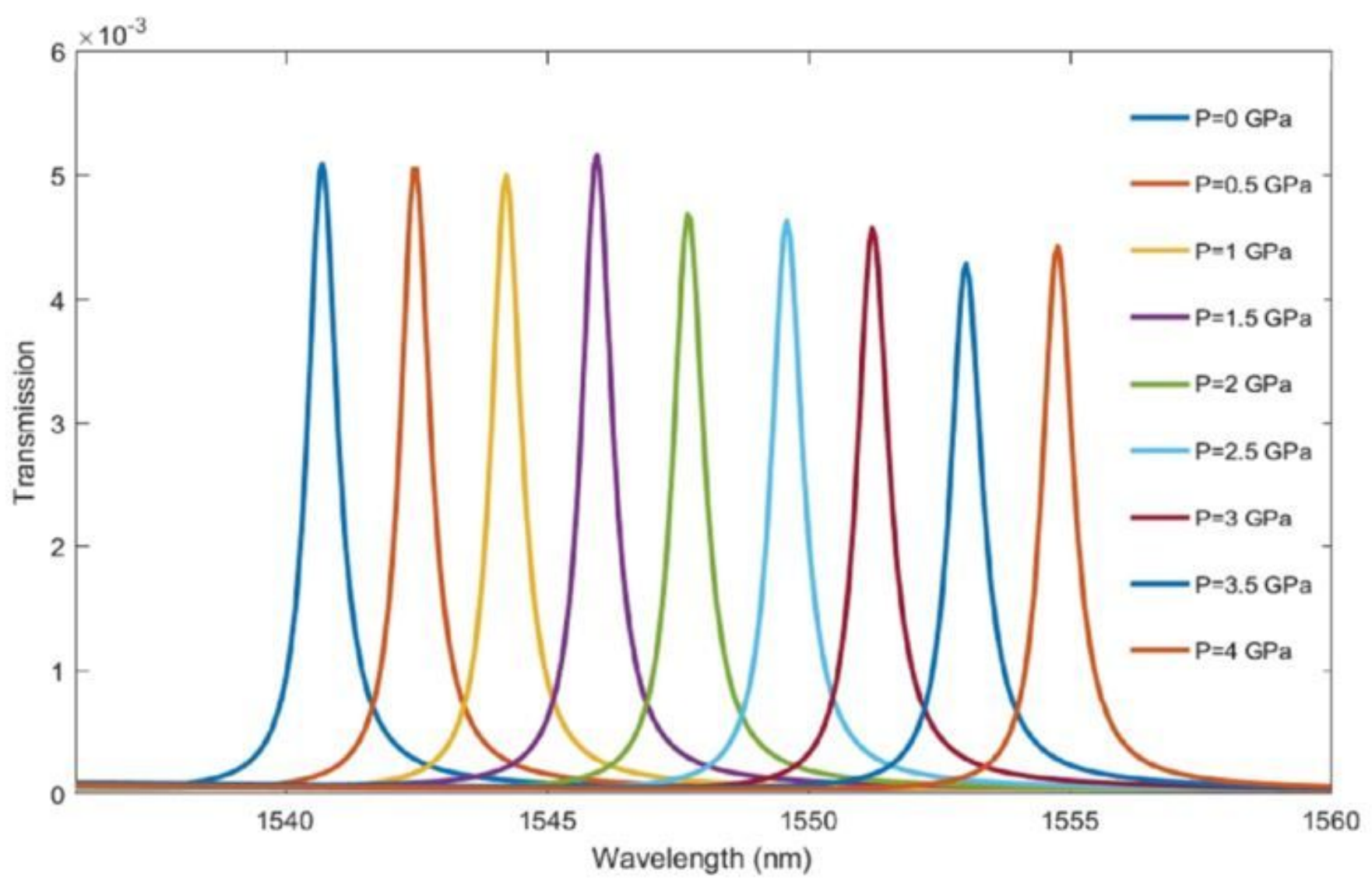

Figure 4

The 3D FDTD simulation results of the transmission spectrum, considering the refractive index variations, for the different values of the applied vertical pressure. 


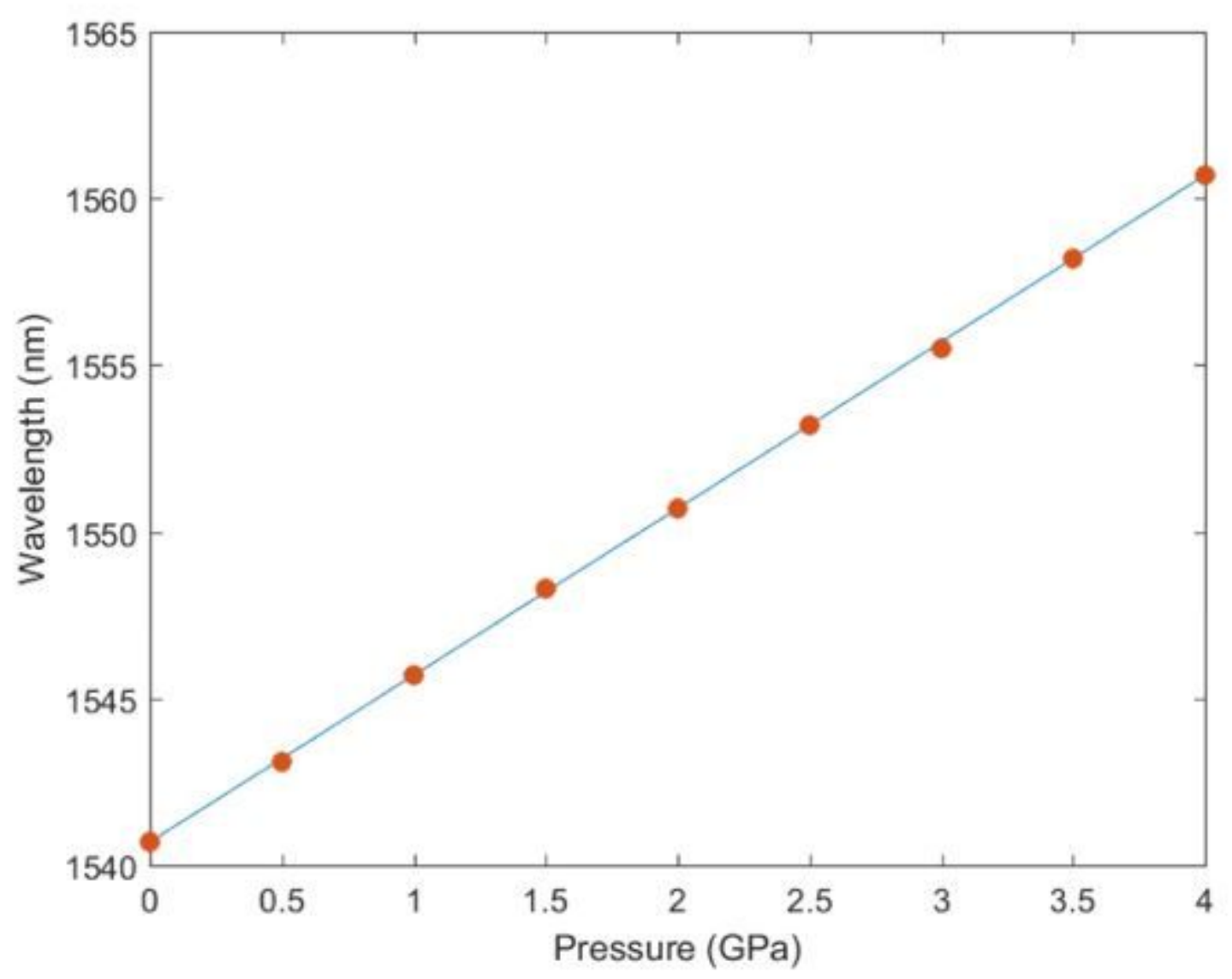

Figure 5

The curve of variations of the resonant wavelength, considering the refractive index variations, as a function of the applied vertical pressure. 

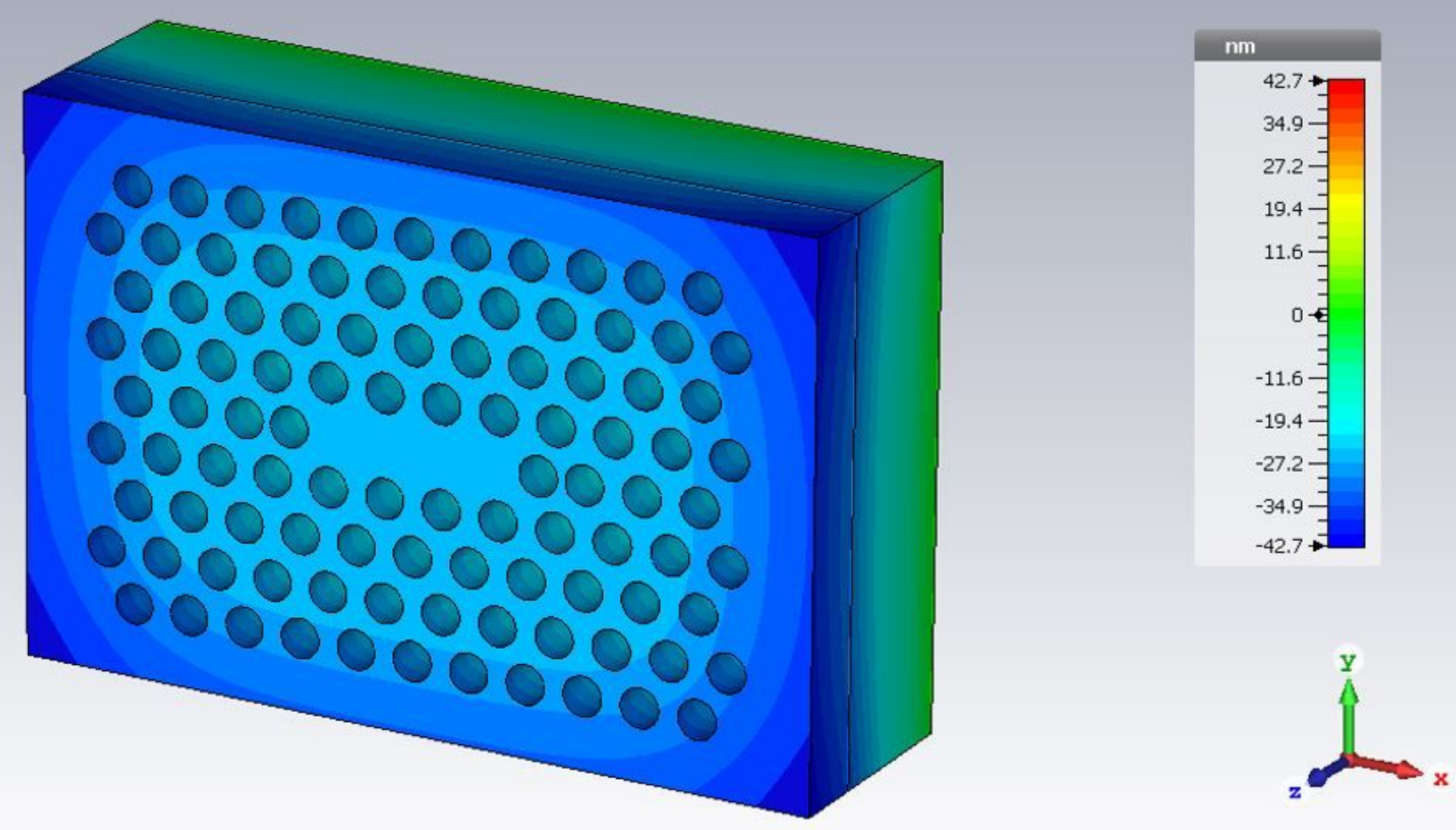

Figure 6

The deformation of the proposed structure when a 4GPa pressure, perpendicular to the plane of incidence (-aa), is applied. 


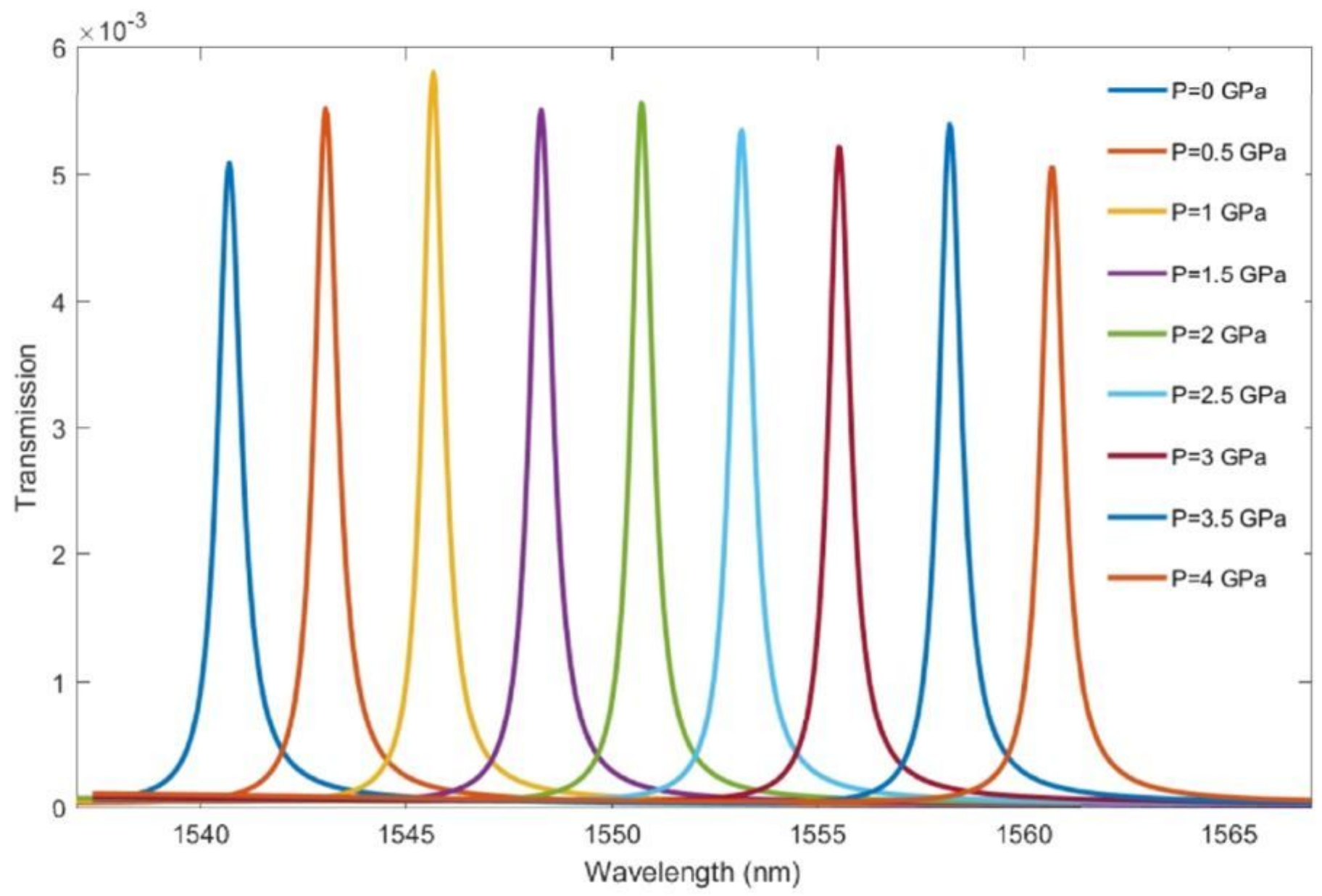

Figure 7

The 3D FDTD simulation results of the transmission spectrum, considering the deformation effect, for the different values of the applied vertical pressure. 


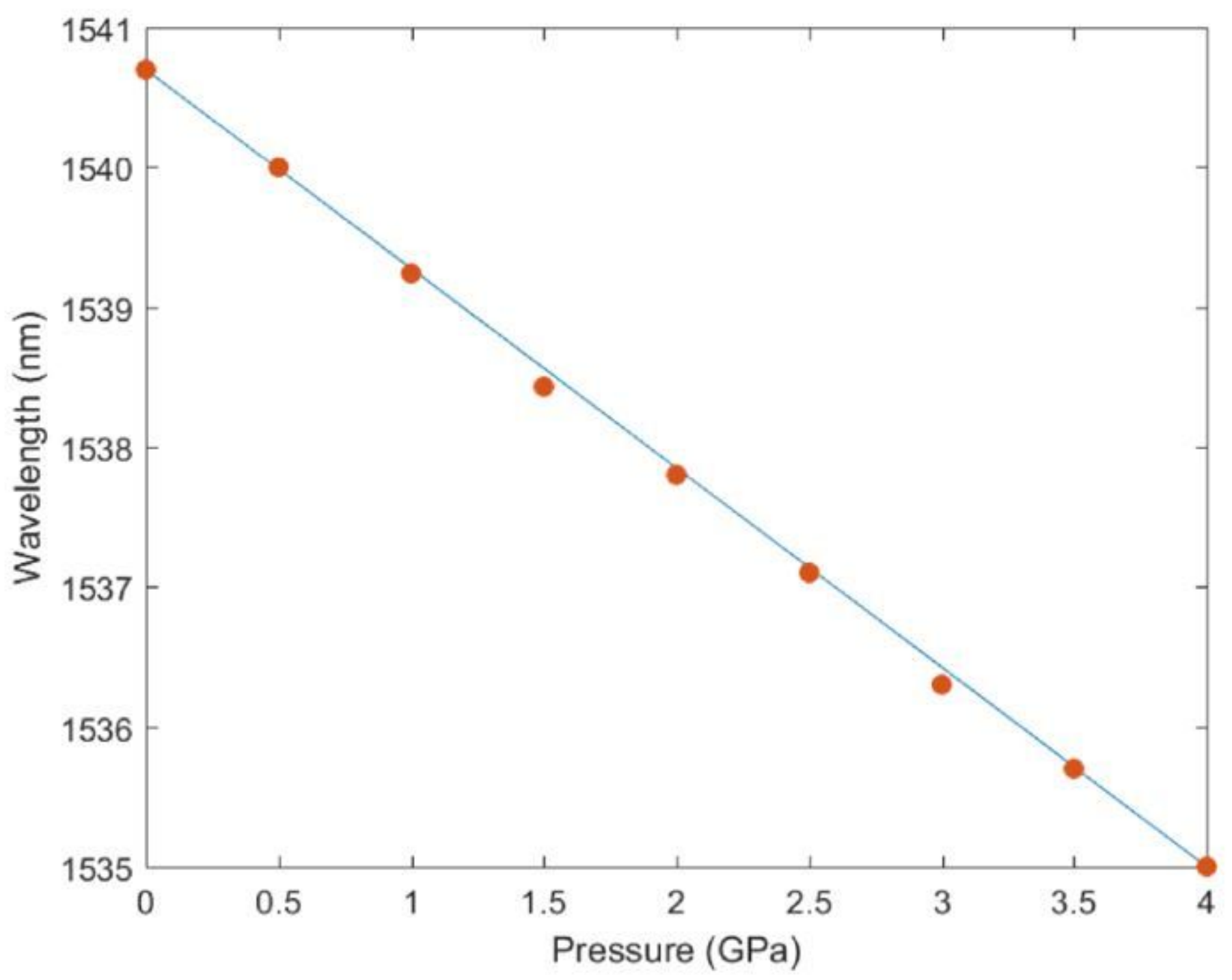

Figure 8

The curve of variations of the resonant wavelength, considering the deformation effect, as a function of the applied vertical pressure. 


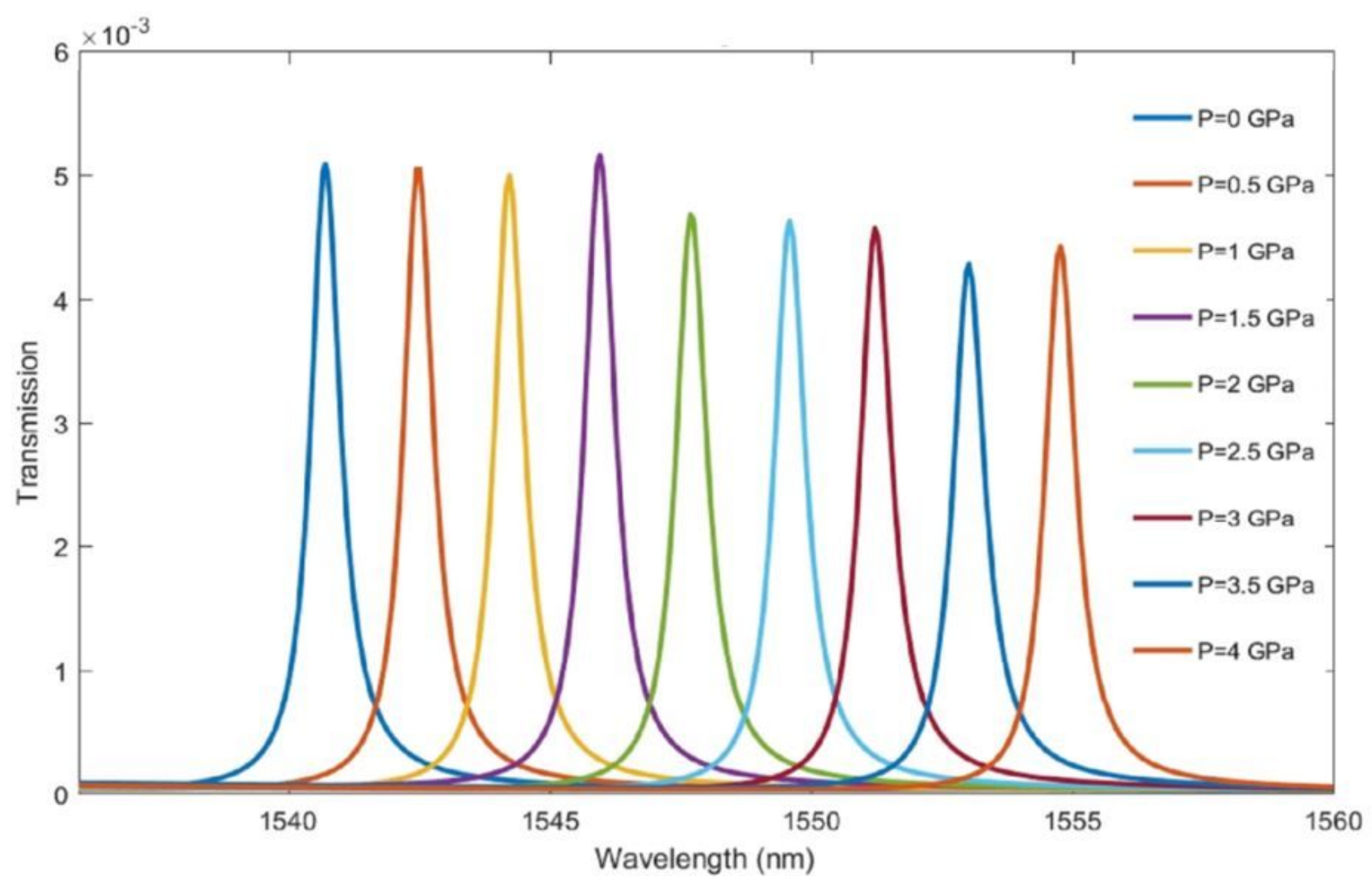

Figure 9

The 3D FDTD simulation results of the transmission spectrum, considering the refractive index variations and the deformation, for the different values of the applied vertical pressure. 


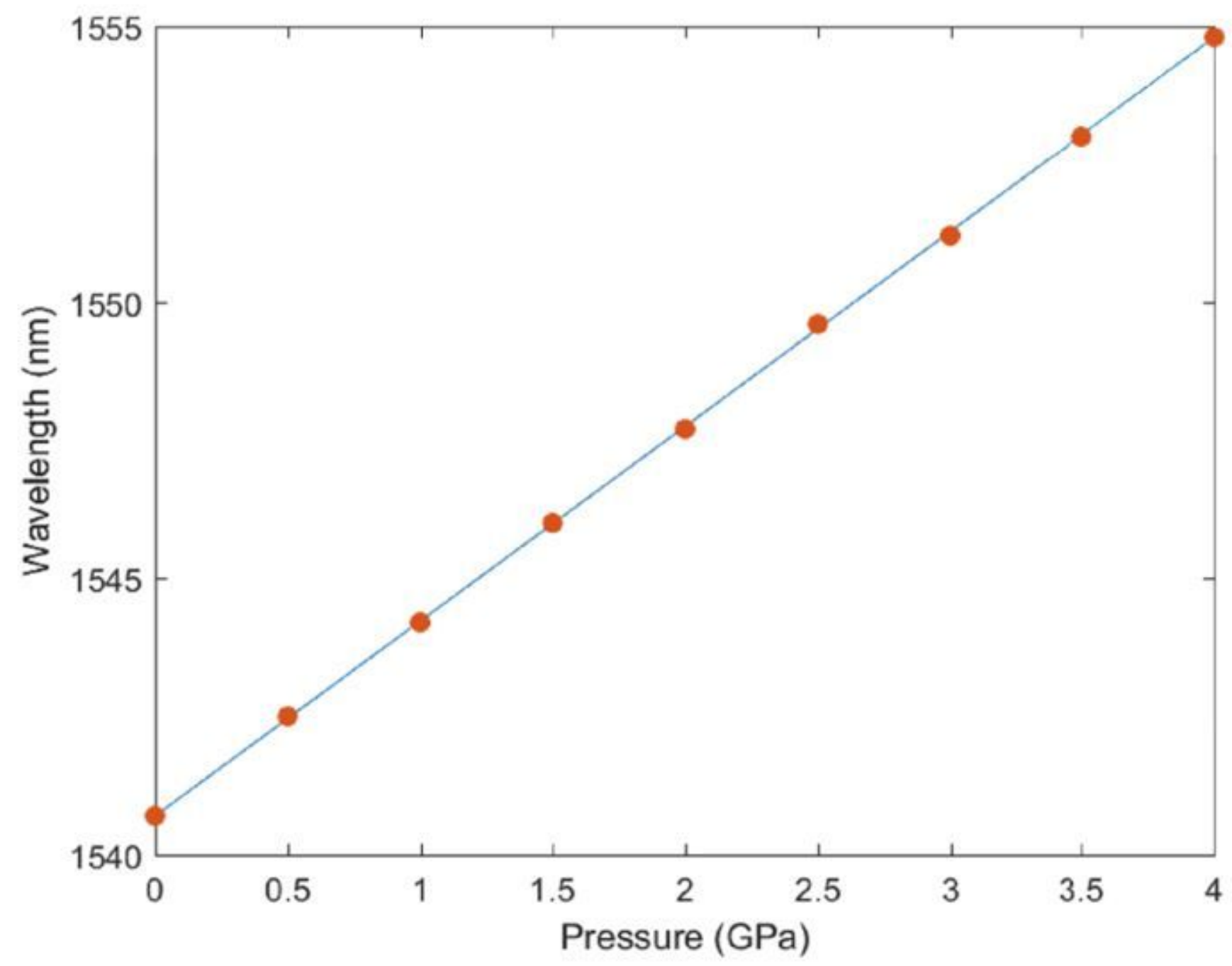

Figure 10

The curve of variations of the resonant wavelength, considering the refractive index variations and the deformation, as a function of the applied vertical pressure. 